\title{
Editorial
}

\section{PET-CT-a Most Advanced Fusion Molecular Imaging Modality of Nuclear Medicine}

\begin{abstract}
H Rahman
Positron emission tomography- Computed tomography (PET-CT) is the most advanced fusion molecular imaging modality of Nuclear Medicine having its unique advantages of both anatomical and physiological information in a single study. PET is called the emission technique where gamma rays created during the emission of positron are detected by the scanner. Positron emitted radionuclides tagged to specific tracers are injected intravenously into the patients. These are then taken up by various organs or tissue either physiologically or pathologically and give an image map that is essential. On the other hand, CT is called the transmission technique where X-ray passes through the body and at the same time from opposite

Since 2015 the National Institute of Nuclear Medicine and Allied Sciences started to perform PET-CT study for the first time in public sector. Now there are three PET-CT facilities available in public and two in private sector in Bangladesh.

Although PET-CT is initially a costly modality for oncology patients in perspective of Bangladesh, still it is very much useful in Bangladesh with less sufferings to patients, better survival rate, avoidance of unnecessary surgery or underuse/overuse of chemotherapy, misuse of radiotherapy and thus making it cost effective and gaining popularity day by day to doctors and patients.
\end{abstract} direction, detection of attenuation of the body is carried out by the detectors and forms an anatomical image. PET and CT are fused on a single gantry into a hybrid technology for the ultimate combination of functional and anatomical image in single frame.

Over the past few decades PET-CT have revolutionized the care of cancer patients in developed countries and are increasingly being adopted in emerging economies. PET-CT has been, and still is, one of the fastest growing fields in medical imaging. It has a powerful synergistic effect that is greater than the sum of the two individual techniques. PET-CT is an indispensable imaging procedure in the management of many different types of cancer. More than $90 \%$ of all PET-CT study is done by ${ }^{18}$ FDG (Fluorodeoxyglucose) for oncology patients for its diagnosis, staging and response to therapy and follow up in the whole world.

Nuclear Medicine is entering a new era with the inclusion of PET-CT in Bangladesh. PET-CT has revolutionized in cancer diagnosis and therapy and a stronger diagnostic tool for the early detection and determines the staging of cancer, therapy planning and follow up. Treatment planning system ( TPS) denotes how much dose is required at the site of the tumor for the treatment of cancer patients before patient undergoes a radiotherapy process. It can precisely determine the site where radiation needs to be focused. In addition, it can give valuable information about the metabolism of myocardium and also useful in diagnosis of many non-malignant diseases.

1. Dr. Hafizur Rahman, MBBS, M Phil (Nuclear Medicine), PMO, Institute of Nuclear Medicine \& Allied Sciences, Faridpur.

Address of correspondence :

Dr. Hafizur Rahman, MBBS, M Phil (Nuclear Medicine), PMO, Institute of Nuclear Medicine \& Allied Sciences, Faridpur.

Mobile:+8801711135280,E-mail: mhm08@yahoo.com 